\title{
Global Progressive Disease in Viscera
}

National Cancer Institute

\section{Source}

National Cancer Institute. Global Progressive Disease in Viscera. NCI Thesaurus. Code C159967.

Greater than 50 percent increase in size by sum of products of diameter (SPD) of any organs involved at baseline; or new organ involvement or loss of response: greater than 50 percent increase from nadir in the size (SPD) of any previous org an involvement in those with partial response (whichever occurs first). 